\title{
THREE-DIMENSIONAL NUMERICAL SIMULATION OF A GAS TUNGSTEN ARC WELDING PROCESS
}

\author{
Karim Agrebi ${ }^{1}$, Asma Belhadj $^{1 *}$, Mahmoud Bouhafs $^{1}$ \\ ${ }^{1}$ Laboratory of Applied Mechanics and Engineering, University of Tunis EL Manar, National \\ Engineering School of Tunis, BP 37, Le Belvédère, 1002, Tunisia
}

(Received: April 2018 / Revised: December 2018 / Accepted: May 2019)

\begin{abstract}
Welding processes are widely used in many industries. The determination of welding parameters and the study of their influence on the mechanical and metallurgical behavior of materials require multiple experiments, and the relevant studies are costly in terms of time and resources. Thus, numerical simulations can serve as a solution when it comes to choosing the appropriate welding process and optimizing its parameters while minimizing costs. The present work contributes to the development of a finite element code, using MATLAB software, for the prediction of thermomechanical and metallurgical behavior during the Tungsten inert gas (TIG) welding process. Numerical computation is based on the mathematical formulation of physical phenomena and thermal exchanges. In this paper, results dealing with the prediction of the temperature field evolution during the C50 steel TIG-welding process are presented. In this case, the thermal problem is solved numerically using the finite element method. The memory and computation time problems are solved using optimal stocking and resolution algorithms. To validate the developed computation code, numerical results are first compared with other published numerical results, then with our experimental data. A satisfactory concordance between simulated temperature evolutions and those measured with thermocouples implanted in the welded sheets was found.
\end{abstract}

Keywords: Finite elements; Mathematical formulation; Thermal simulation; TIG welding

\section{INTRODUCTION}

During welding, highly complicated phenomena occur due to the coupled interactions between heat transfer, metallurgical transformation, and mechanical behavior. To be able to predict the evolution of these phenomena, numerical methods - especially the finite element method (FEM) - are widely used. This method, which is performed using calculation codes like TRANSWELD (Hamide \& Bellet, 2007; Hamide et al., 2008), ASTER CODE (Delmas, 2013) and SYSWELD, is based on mathematical formulation of the coupled physical phenomena.

Several works have been published on numerical simulation of the welding process. Simulating thermal behavior during welding processes is based on numerically solving three-dimensional transient heat equations with temperature-dependent material properties (Belhadj et al., 2010; Anca et al., 2011; Seleš et al., 2018). Numerical calculations require a longer time calculation, high storage capacity, and significant computer resources to predict the thermal, followed by metallurgical and mechanical, history. For this reason, using an adaptive mesh can be a solution for solving calculation problems (Hamide \& Bellet, 2007; Hamide et al., 2008).

\footnotetext{
${ }^{*}$ Corresponding author's email:asmabelhadj2011@yahoo.fr, Tel. +216-21-025081, Fax. +216-71-872729
} Permalink/DOI: https://dx.doi.org/10.14716/ijtech.v10i4.1849 
For the validation of thermal simulation results during welding processes, researchers have compared temperature evolutions with experimental data, analytical solutions, or simulation results with other FEM code calculation. Anca et al.(2011) compared simulated temperature evolutions during welding processes with a semi-analytical solution developed by Weiner and Boley (1963). Moreover, Belhadj et al.(2010) simulated thermal behavior during $\mathrm{CO}_{2}$ laser welding and compared the temperature evolution according to time with the thermocouplemeasured temperature in many positions on a welded sheet.

The TIG welding process is one of the most common methods used in the aerospace, automotive, and pipe industries (Lundbäck, 2003; Baskoro et al., 2011).TIG process welding, especially of stainless steel, has been studied numerically and experimentally by many authors (Del Coz Diaz et al., 2010; Ganesh et al., 2014; Aissani et al., 2015; Chuaiphan \& Srijaroenpramong, 2018). These numerical simulations have been developed with software like ABAQUS, ASTER CODE, and ANSYS. These software programs offer a significant computing potential, especially for thermo-mechanical modeling. However, they are limited for modeling other phenomena occurring during the welding of some materials, such as metallurgical transformations or convection flow in the melt. Nevertheless, these limitations can be overcome via the user's ability to develop and integrate functions, employing subroutines to extend the possibilities of this software and providing the flexibility required for any research work. In this study, we have opted for the development of a specific numerical calculation tool, integrating all the welding phenomena, using the MATLAB software to simulate material behavior during the TIG welding of phase transformation steel. This computer code gives the possibility to predict the thermomechanical and metallurgical transformations in the welded sheets from the beginning of the welding until the end of the cooling.

In this paper, thermal history prediction and its validation are presented. A thermal model with a moving heat source is developed to calculate the temperature on each element of an adaptive mesh during the welding and cooling stages. The developed thermal model considers the nonlinearity introduced by thermo-physical properties, which depends on the temperature. The numerical results are presented in the form of isotherms at different times of welding and temperature evolutions according to time in each point of the mesh. In addition to numerical model development, an experimental protocol is performed to optimize the TIG welding parameters of the C50 steel, realize automatic welding lines, and specifically, measure temperature data during welding using K-thermocouples implanted in several points on the welded sheet. These experimental results aim to validate the numerical results from the developed model. Therefore, finite element simulated temperature evolutions according to time are compared with those measured experimentally.

\section{EXPERIMENTAL METHODS}

\subsection{Modeling of Thermal Behavior during TIG Welding}

\subsubsection{Assumptions}

During welding, thermal, metallurgical, and mechanical phenomena occur and interact to provide the final physical characteristics of the welded parts. In this study, we consider only the thermal phenomena during welding, and two assumptions are made to formulate the problem. The assumptions are as follows:

- Heat transfer due to metallurgical transformation or mechanical evolution is neglected; and

- Convective exchanges in the fusion zone are neglected. 


\subsubsection{Mathematical Formulation}

During welding, the welded sheet receives movable and localized heat energy on $\partial \Omega_{T I G}$, where its temperature increases to the melting point.The heat propagates by conduction in the piece volume $\Omega$ and convection and radiation on the sheet surface $\partial \Omega$.Thermal exchanges during the TIG welding process are illustrated by Figure 1.

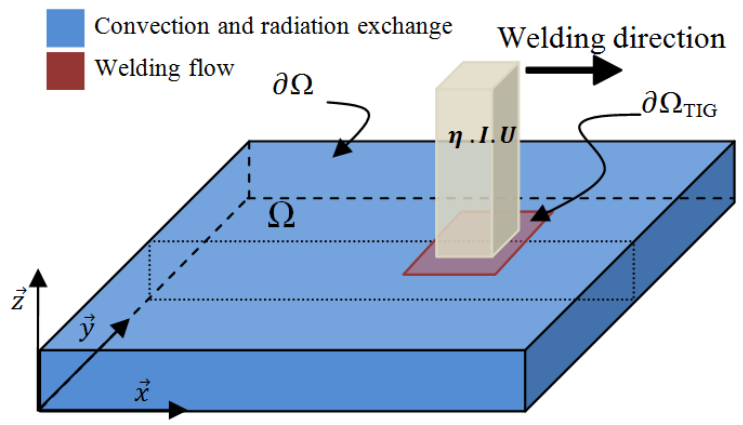

Figure 1 Heat transfer during welding

The thermal problem is governed by the energy balance equation using the enthalpy method, considering the phase change(Belhadj et al., 2010). The local form of the energy balance equation is given by Equation 1:

$$
\nabla(\lambda(T) \vec{\nabla} T)=\rho(T) \cdot \frac{d H}{d t} \quad \forall M \in \Omega,
$$

where $T$ is the temperature, $\lambda(T)$ is the material thermal conductivity depending on temperature, and $\rho(T)$ is the materialdensitydepending on temperature. In addition, His the specific enthalpy, which is given by Equation2:

$$
H(T)=\int_{T_{0}}^{T} C_{p}(\tau) \cdot d \tau+g_{l} \cdot L_{f},
$$

Where $T_{0}$ is the initial temperature, $C_{p}$ is the specific heat, $L_{f}$ is the specific fusion latent heat and $g_{l}$ is the fraction of liquid given by Equation 3 .

$$
\begin{aligned}
& g_{l}(T)=0 \quad \text { if } T<T_{S} \\
& g_{l}(T)=\frac{T-T_{S}}{T_{l}-T_{S}} \quad \text { if } T_{S} \leq T \leq T_{l} \text {, } \\
& g_{l}(T)=1 \quad \text { if } T_{l}<T
\end{aligned}
$$

where $T_{S}$ is the solidus temperature and $T_{l}$ is the liquidus temperature.

Boundary conditions describing the heat exchange between the welded sheet and the ambient air are:

- The convection heat transfer is coverned by Equation 4 .

$$
-\lambda \cdot \vec{\nabla} T \cdot \vec{n}=h .\left(T-T_{\text {ext }}\right) \text { on } \partial \Omega,
$$

where $h$ is the convection coefficient, $T_{\text {ext }}$ is the ambient temperature and $\vec{n}$ is the outward normal unit vector.

- The radiation heat transfer is covered by Equation 5.

$$
-\lambda \cdot \vec{\nabla} T \cdot \vec{n}=\varepsilon \cdot \sigma \cdot\left((T+273)^{4}-\left(T_{e x t}+273\right)^{4}\right) \text { on } \partial \Omega,
$$

where $\varepsilon$ is the material emissivity and $\sigma$ is the Stefan-Boltzmann constant.

The heat source is modeled with a flux density uniformly distributed over a square task. The thermal power of welding during TIG welding is simulated by Equation 6 . 


$$
-\lambda \cdot \vec{\nabla} T \cdot \vec{n}=q_{T i g} \quad \text { on } \partial \Omega_{T I G},
$$

where $q_{T i g}$ is the imposed surface heat flow given by Equation 7 .

$$
q_{\text {Tig }}=\frac{\eta \cdot I \cdot U}{a^{2}},
$$

where $U$ is the welding voltage, $I$ is the welding current, $\eta$ is the arc station performance and a is the square task dimension.

The initial condition is the sheet temperature before starting the welding given by Equation 8 .

$$
T(M, 0)=T_{0}{ }^{\circ} \mathrm{C} \quad \forall(M) \in \Omega .
$$

This Mathematical formulation will be developed, using finite elements method, in order to predict numerically the temperature evolution during gas tungsten arc welding.

\subsection{Finite Element Formulation}

\subsubsection{Spatial discretization}

The welded piece is subdivided into hexagonal finite elements,using the mesh in eight knots(Figure 2).The mesh is refined approaching the fusion line and along the transverse axis.This technique can make a dense mesh moving simultaneously with the heat source, while the other area of the structure has a much coarser mesh, greatly reducing the number of nodes and elements in the analysis. In the spring-based method, the edges between any two mesh nodes are linked by a network of interconnected springs like shown in Figure3.The initial spacings of the edges constitute the equilibrium state of the mesh.when moving the heat source we change the spring stiffness value and recalculate the new equilibrium.

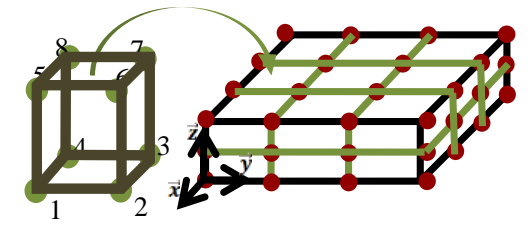

Figure 2 Space discretization

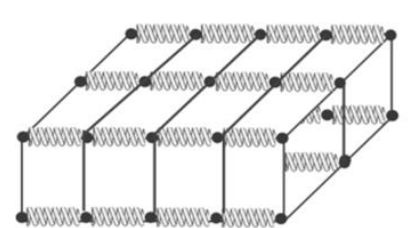

Figure 3Spring-based method for adaptive mesh

The mesh adaptation is done along the welding axis $\vec{x}$. For the axis $\vec{y}$ and $\vec{z}$, we used a fixed mesh not uniformwhich becomes greater far away from the welding line.The configuration of the adaptatif mesh for different position of the heat source and after the exit of this one is shown in Figure 4.
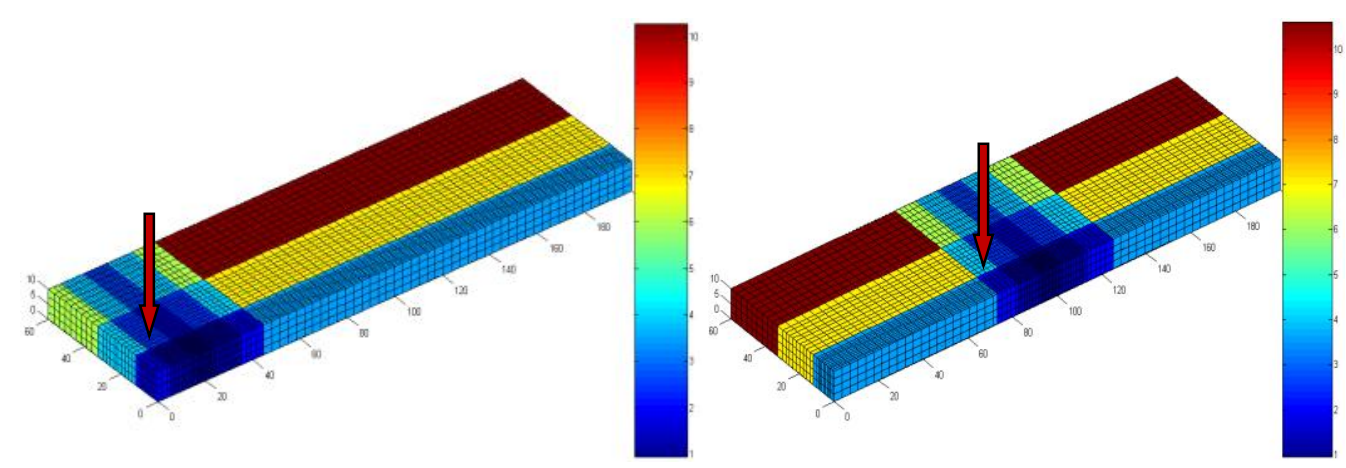

Figure 4 Adaptive mesh construction during welding

\subsubsection{Variational formulation for thermal problem}

Using Equations 1, 2, variational formulation for the thermal problem is given by Equation 9 . 


$$
-\int_{\Omega} \lambda \cdot \vec{\nabla} T \cdot \vec{\nabla} T^{*} d v+\int_{\partial \Omega} \lambda \cdot \vec{\nabla} T \cdot \vec{n} \cdot T^{*} d s=\int_{\Omega} \rho \cdot C_{p}(T) \cdot \frac{\partial T}{\partial t} \cdot T^{*} \cdot d \Omega+\int_{\Omega} \rho \cdot L_{f} \cdot \frac{\partial g_{l}}{\partial t} \cdot T^{*} \cdot d \Omega .
$$

Taking into account the boundary conditions given by Equations 4, 5, the variational formulation can be written by Equation 10 .

$$
\begin{gathered}
-\int_{\Omega} \lambda \cdot \vec{\nabla} T \cdot \overrightarrow{g r a d} T^{*} d \Omega-\int_{\partial \Omega} h \cdot\left(T-T_{\text {ext }}\right) \cdot T^{*} d s-\int_{\partial \Omega} \varepsilon \cdot \sigma \cdot\left((T+273)^{4}-\left(T_{\text {ext }}+273\right)^{4}\right) \cdot T^{*} d s+ \\
\int_{\partial \Omega_{T I G}} q_{T i g} \cdot T^{*} d s=\int_{\Omega} \rho \cdot C_{p}(T) \cdot \frac{\partial T}{\partial t} \cdot T^{*} \cdot d v+\int_{\Omega} \rho \cdot L_{f} \cdot \frac{\partial g_{l}}{\partial t} \cdot T^{*} \cdot d v .
\end{gathered}
$$

In the finite element method, the unknown temperature field $T$ is calculated approximaty as a linear combination of interpolation functions $\Phi_{j}(M)$, and given by Equation 11.

$$
T(M, t)=\sum T_{j} . \Phi_{j}(M) .
$$

where $T_{j}$ is the temperature at each node $j(\mathrm{j}=1,2, \ldots, 8)$ from $\Omega$ and $\Phi_{\mathrm{j}} 1 \leq j \leq$ 8are the Lagrange function.

The thermal elementary system is given for each finite element of mesh(Equation12). Rigidity matrix and force vector are given respectively by Equations 13, 14.

$$
\begin{gathered}
\left\{\begin{array}{c}
{[T]^{1}=\left[T_{0}\right]} \\
{\left[K_{e l}\right]_{k}^{n} \cdot[T]^{n+1}=\left[F_{e l}\right]_{k}^{n} \cdot}
\end{array}\right. \\
{\left[K_{e l}(i, j)\right]_{k}^{n}=\lambda_{k}^{n} \cdot \int_{e_{k}} \vec{\nabla} \Phi_{j} \cdot \vec{\nabla} \Phi_{i} \cdot d \Omega-h \cdot \int_{\partial e_{k}^{c o n v}} \Phi_{j} \cdot \Phi_{i} d s-\frac{1}{d t} \cdot \rho_{k}^{n} \cdot\left(C_{p}\right)_{k}^{n} \cdot \int_{e_{k}} \Phi_{j} \cdot \Phi_{i} \cdot d \Omega .} \\
{\left[F_{e l}(i, j)\right]_{k}^{n}=-h \cdot T_{e x t} \cdot \int_{\partial e_{k}^{c o n v}} \Phi_{i} d s+\varepsilon \sigma\left(\left(T_{k}^{n}+273\right)^{4}-\left(T_{e x t}+273\right)^{4}\right) \cdot \int_{\partial e_{k}^{r a y}} \Phi_{i} d s} \\
-q_{T i g} \int_{\partial e_{k}^{T I G}} \Phi_{i} d s-\frac{1}{d t} \cdot \rho_{k}^{n} \cdot\left(C_{p}\right)_{k}^{n} \cdot \int_{e_{k}} \Phi_{j} \cdot \Phi_{i} \cdot d \Omega+. \rho_{k}^{n} \cdot\left(\dot{g}_{l}\right)_{k}^{n} \cdot \int_{e_{k}} \Phi_{i} \cdot d \Omega .
\end{gathered}
$$

where $n$ is the previous iteration and the $n+1$ actually iteration, $[\mathrm{T}]^{\mathrm{n}}$ is the nodal temperature vector at $\mathrm{n}$-thiteration, $\mathrm{T}_{\mathrm{k}}^{\mathrm{n}}$ is the average temperature of the $\mathrm{k}$-number element at the $\mathrm{n}$-thiteration, $\left[\mathrm{F}_{\mathrm{el}}\right]_{\mathrm{k}}^{\mathrm{n}}$ is the elementary force vector for on the k-number element at the $\mathrm{n}$-th iteration, $\left[\mathrm{K}_{\mathrm{el}}\right]_{\mathrm{k}}^{\mathrm{n}}$ is the elementary rigidity matrix for the k-number element at the $n$-th iteration, $\left(C_{p}\right)_{k}^{n}$ is the average value of heat capacity on the k-number element at the $n$-th iteration, $\rho_{\mathrm{k}}^{\mathrm{n}}$ is The average density on the $\mathrm{k}$-number element at the $\mathrm{n}$-th iteration, $\lambda_{\mathrm{k}}^{\mathrm{n}}$ is the average value of heat conductivity on the knumber element at the $n$-th iteration, $\partial \mathrm{e}_{\mathrm{k}}^{\mathrm{TIG}}$ is $\mathrm{k}$-number element face which exchanges the TIG source heat, $\partial \mathrm{e}_{\mathrm{k}}^{\text {conv }} \mathrm{k}$-number element faces which exchanges the convection heat and $\partial \mathrm{e}_{\mathrm{k}}^{\text {ray }}$ is $\mathrm{k}$ number element faces which radiation heat.

The elementary systems already constructed will be assembled in one system. Theassembly operation is done by applying Equations 15-16.

$$
\begin{aligned}
& K_{g}(i, j)=\sum_{k=1}^{n b e l} \sum_{u=1}^{8} \sum_{v=1}^{8}\left[K_{e l}(u, v)\right]_{k} / i=\operatorname{con}(k, u) \text { and } j=\operatorname{con}(k, v) . \\
& F_{g}(i)=\sum_{k=1}^{n b e l} \sum_{u=1}^{8}\left[F_{e l}(u)\right]_{k} / i=\operatorname{con}(k, u) .
\end{aligned}
$$

where $\left[\mathrm{K}_{\mathrm{el}}\right]_{\mathrm{k}}$ and $\left[\mathrm{F}_{\mathrm{el}}\right]_{\mathrm{k}}$ are respectively the elementary rigidity matrix and the elementary force vector of the $\mathrm{k}$-number element, $\mathrm{K}_{\mathrm{g}}$ and $\mathrm{F}_{\mathrm{g}} \mathrm{F}_{\mathrm{g}}$ are respectively the global rigidity matrix and the global force vector, con(.,.) is the mesh connection matrix andn bel $_{\text {is }}$ ise element number in the mesh.

The rigidity matrixes, given by Equation 17, areobtained by finite elements method with application of the band form of sparse matrix. In order to minimize the stocking memories; we conserve only the non-zero elements. We adopt a stocking form which consists in writing rigidity 
matrix lines successively one after one in a vector and we conserve only the nonzero element inside the band. The rigidity matrix lines are given by Equation 18 .

$$
\begin{gathered}
K_{g}=\left[\begin{array}{ccccccc}
K_{11} K_{12} & \ldots & K_{1 a} & & & & \\
\vdots & \ddots & \vdots & \ddots & & 0 & \\
K_{a 1} & \ldots & K_{a a} & & & & \\
& \ddots & & \ddots & & \ddots & \\
& & & & K_{r-a r a} a & \ldots & k_{a r r} \\
& 0 & & \ddots & \vdots & \ddots & \vdots \\
& & & & k_{r r-a} & \ldots & k_{r r}
\end{array}\right] . \\
V K_{g}=\left[\begin{array}{llllll}
K_{11} K_{12} & \ldots & K_{1 a} K_{2 a} & \ldots & K_{r r}
\end{array}\right] .
\end{gathered}
$$

\subsubsection{Finite Element systems resolution}

The numerical system is solved using Gauss triangulation method consisting in the factorization of the stiffness matrix in two upper and lower triangular matrix.This Finite Element formulation will be programmed using MATLAB Software in order to simulatetemperature evolution during TIG welding.

\subsection{Finite Element Simulation}

\subsubsection{Simulation assumptions}

For the numericalcalculation, only one-half of the plateis analyzed due to symmetry of the weld. So for this reason, adiabatic boundary conditions are defined on the surface symmetry.

\subsubsection{Simulation parameters}

To predict temperature evolution during welding using F-E calculation, we must have mesh characteristics, Welded materiel properties according temperature, TIG Welding parameters and heat exchange characteristics.

In this work, the finite element geometric model is an adaptive meshpresented in Figure 5. The mesh consists of 21816 nodes and 17500 linear hexahedral elements. The calculationtime step is $1 \mathrm{~s}$.

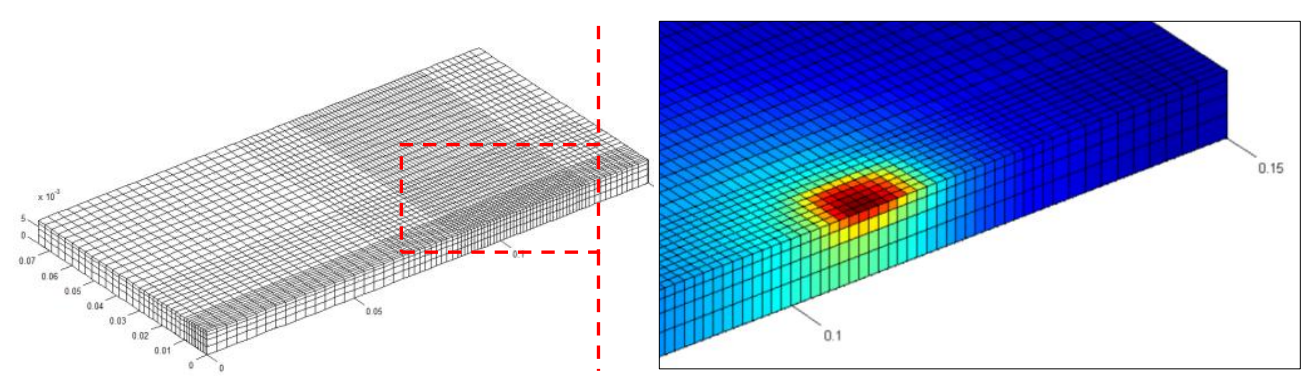

Figure 5 Adaptive mesh used in TIG Welding FEM simulation

The based material used in this investigation is the EN C50 steel. Thermo-physical characteristics of this material, depending on temperature, are shown in Table 1.

Table 1Thermo-physical properties of C50 steel

\begin{tabular}{lcccccc}
\hline $\boldsymbol{T}\left({ }^{\circ} \boldsymbol{C}\right)$ & $\mathbf{2 0}$ & $\mathbf{1 0 0}$ & $\mathbf{2 0 0}$ & $\mathbf{3 0 0}$ & $\mathbf{4 0 0}$ & $\mathbf{5 0 0}$ \\
$\lambda(\mathrm{W} / \mathrm{m} . \mathrm{K})$ & 52 & 50 & 47 & 45 & 42 & 38 \\
$\rho\left(\mathrm{Kg} / \mathrm{m}^{3}\right)$ & 7850 & 7750 & 7800 & 7780 & 7740 & 7690 \\
$\mathrm{Cp}(\mathrm{J} / \mathrm{Kg} . \mathrm{K})$ & 460 & 480 & 500 & 515 & 530 & 570 \\
\hline $\boldsymbol{T}\left({ }^{\circ} \boldsymbol{C}\right)$ & $\mathbf{6 0 0}$ & $\mathbf{7 0 0}$ & $\mathbf{8 0 0}$ & $\mathbf{9 0 0}$ & $\mathbf{1 0 0 0}$ & \\
$\lambda(\mathrm{W} / \mathrm{m} . \mathrm{K})$ & 34 & 30 & 24 & 25 & 27 & \\
$\rho\left(\mathrm{Kg} / \mathrm{m}^{3}\right)$ & 7650 & 7630 & 7620 & 7580 & 7520 & \\
$\mathrm{Cp}(\mathrm{J} / \mathrm{Kg} . \mathrm{K})$ & 600 & 630 & 675 & 675 & 675 & \\
\hline
\end{tabular}


Table 2 and Table 3 give respectively welding and thermal exchange parameters used in the numerical simulation of the thermal history during TIG welding. Welding parameters given in Table 3 are those used for the experimental investigations.

Table 2TIG Welding parameters

\begin{tabular}{lll}
\hline Welding speed & $v$ & $0.72 \mathrm{~mm} / \mathrm{s}$ \\
Welding current & $I$ & $100 \mathrm{~A}$ \\
Welding voltage & $U$ & $15.2 \mathrm{~V}$ \\
Square task dimension & $a$ & $10 \mathrm{~mm}$ \\
Arc station performance & $\eta$ & 0.58 \\
\hline
\end{tabular}

Table 3Thermal exchange parameters

\begin{tabular}{lcl}
\hline Solidus temperature & $T_{S}$ & $1400{ }^{\circ} \mathrm{C}$ \\
Liquids temperature & $T_{l}$ & $1450{ }^{\circ} \mathrm{C}$ \\
Fusion latent heat & $L_{f}$ & $2.42105 \mathrm{~J} / \mathrm{kg}$ \\
Initial temperature & $T 0$ & $20{ }^{\circ} \mathrm{C}$ \\
Convective coefficient & $h$ & $5 \mathrm{~W} /(\mathrm{m} 2 \mathrm{~K})$ \\
Emissivity coefficient & $\varepsilon$ & 0.3 \\
\hline
\end{tabular}

\subsection{Experimental Investigations}

Experimental tests were conducted to collect data on temperature field on the welded sheet. The experimental results will be used to verify the finite elements simulation results.A line welding is done on a C50 steel $7 \mathrm{~mm}$ thickness plate using an automatic TIG welding station (Figure 6). Thermal source is moving at $0.72 \mathrm{~mm} / \mathrm{s}$ along $\vec{x}$ axis. Welding current and arc voltage are respectively $\mathrm{I}=100 \mathrm{~A}$ and $\mathrm{U}=15.2 \mathrm{~V}$ and the arc station performance is $\eta=0.58$. The welding arc starts at $7 \mathrm{~mm}$ from the edge of the plate and ends in the middle of the plate at the same level with those thermocouples. To record temperature evolution during TIG welding process, K-type thermocouples were used. As shown in Figure 7, thermocouples were fixed near the weld at two different distances to the weld centre-line on upper face (P1) and lower face (P2, P3) of the welded sheets.

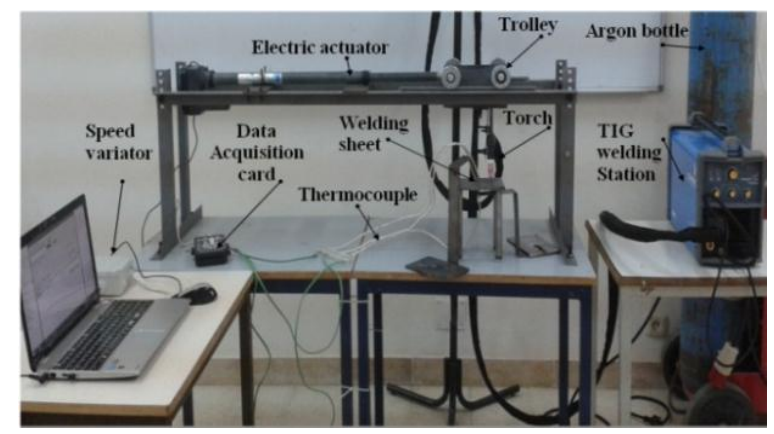

Figure 6 Welding investigation

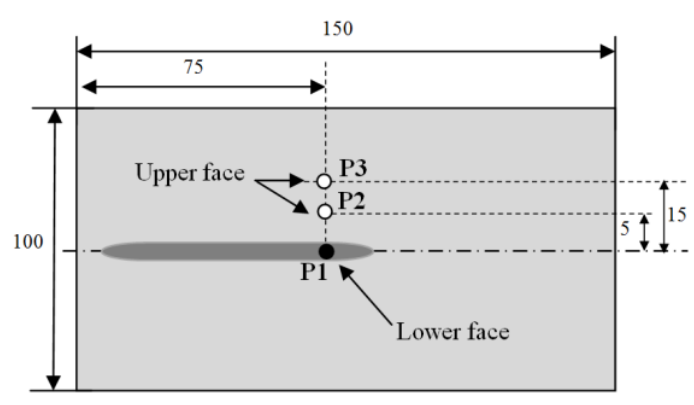

Figure7 K-type thermocouples position

\section{RESULTS AND DISCUSSION}

\subsection{Numerical Results}

The developed FM code gives possibility to predict thermal behavior during C50 TIG welding and cooling.Simulation results are presented in form of space and time temperature evolution.Figure 8 shows temperature evolution in several positions in the welded sheets. Figure 9 illustrates temperature space distributions from the beginning of welding to the cooling phase.The temperature evolution according time in each node of the mesh can be also predicted. 

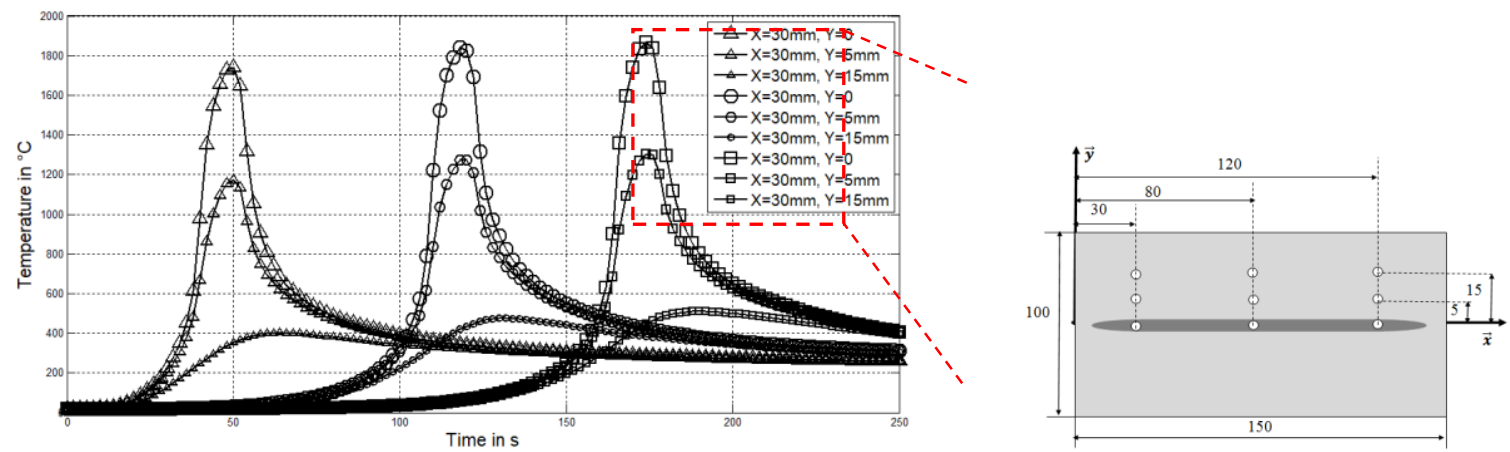

Figure 8 Simulated temperature evolutions during welding in several positions in the welded sheets

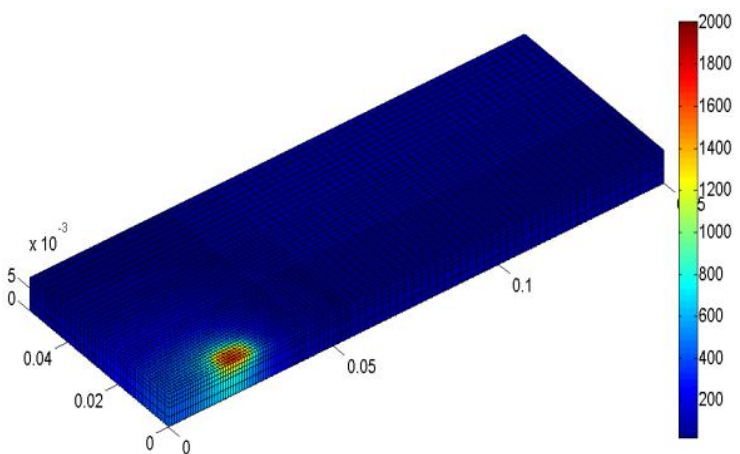

After36s welding

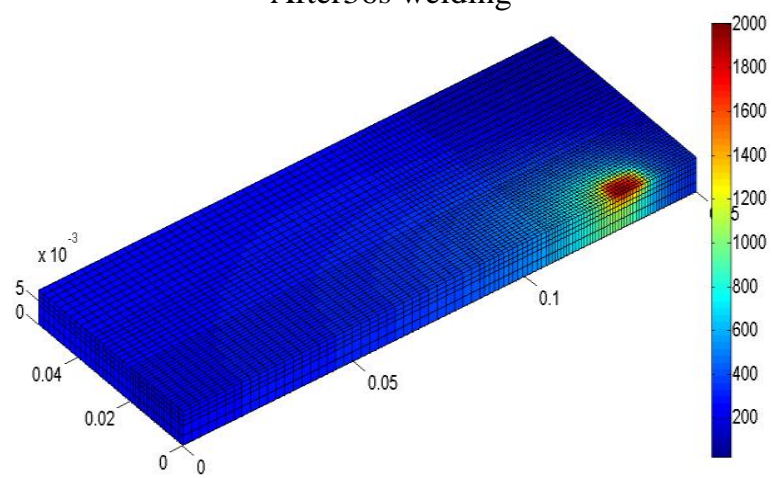

After190s welding

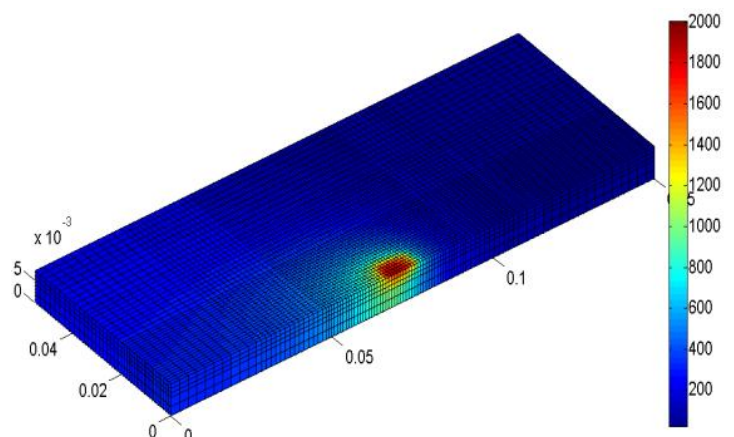

After106s welding

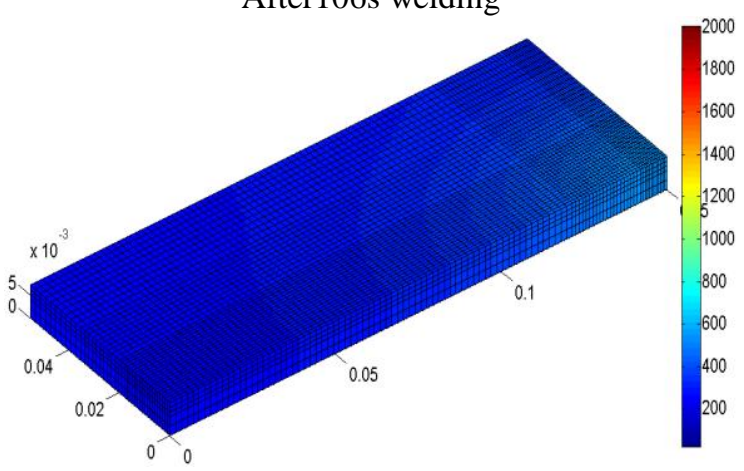

After240s welding (cooling phase)

Figure 9 Temperature field during the welding and the cooling phases for a speed welding $v=0.72 \mathrm{~mm} / \mathrm{s}$

\subsection{Experimental Results}

Temperature evolutions are measured with three thermocouples implanted in the welded sheet during TIG welding (Figure 7). From measured temperature evolution shown in Figure 10, we can note that there are two stages of the process: the heating one for which the heating rate is important compared to quenching rate during cooling stage.

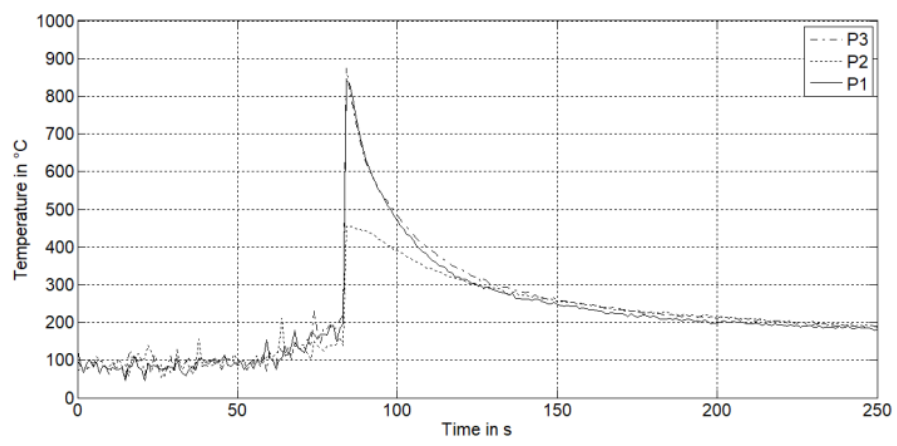

Figure 10 Measured temperature according time in different points of the welded sheet for $\mathrm{v}=0.72 \mathrm{~mm} / \mathrm{s}$ 


\subsection{Numerical Validation of Developed FE Code}

The finite element calculated temperatures in this work are compared to the temperature evolution resulting from other numerical calculationlike those madeby Depradeux and Jullien (2004). Using ASTER code, these authors simulate the thermal problem as a line of fusion carried out by TIG welding on a316 L stainless steel plate. A line welding is done on a $10 \mathrm{~mm}$ thickness plate using TIG welding station. Welding current and arc voltage are respectively $\mathrm{I}=150 \mathrm{~A}$ and $\mathrm{U}=10 \mathrm{~V}$. Arc station performance is $\eta=0.68$. In our work, Heat source is modeled by a squared surface heat distribution with $10 \mathrm{~mm}$ side. Simulated thermal source is moving at $1 \mathrm{~mm} / \mathrm{s}$ and $0.67 \mathrm{~mm} / \mathrm{s}$ along $\vec{x}$ axis. Heat exchange is made by convection and radiation. The convection exchange coefficient is $\mathrm{h}=5 \mathrm{~W} / \mathrm{m}^{2} . \mathrm{K}$ and emissivity coefficient is $\varepsilon=0.75$. These welding parameters and the $316 \mathrm{~L}$ stainless steel characteristics are used to calculate the numerical temperature evolution with our developed code. According to Figure 11, the comparison of our finite element simulated temperature evolution and those obtained by ASTER Code reveals a good agreement between results. We can note that our developed code gives good results for TIG material with other materials welded with many welding speeds.
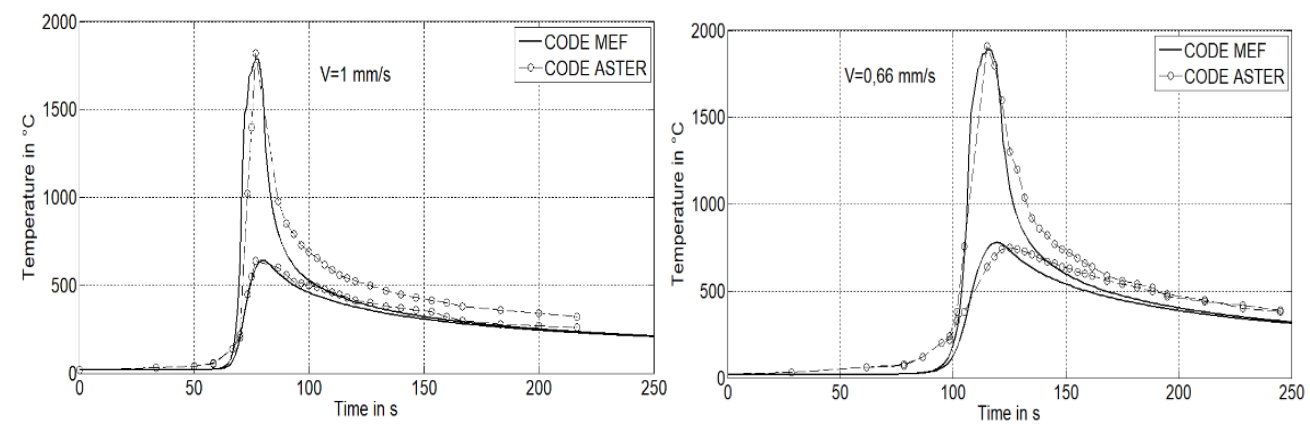

Figure 11 Comparison of simulated temperature evolutions with developed code and ASTER code (Depradeux \& Jullien, 2004) for welding speeds $\mathrm{v}=1 \mathrm{~mm} / \mathrm{s}$ and $\mathrm{v}=0.67 \mathrm{~mm} / \mathrm{s}$

\subsection{Experimental Validation of FEM Developed Code}

Experimental investigations are used to validate the FEM developed code results. Numerical temperature evolutions are compared to those measured by thermocouples fixed in three points of the welded sheets and shown in Figure 12.

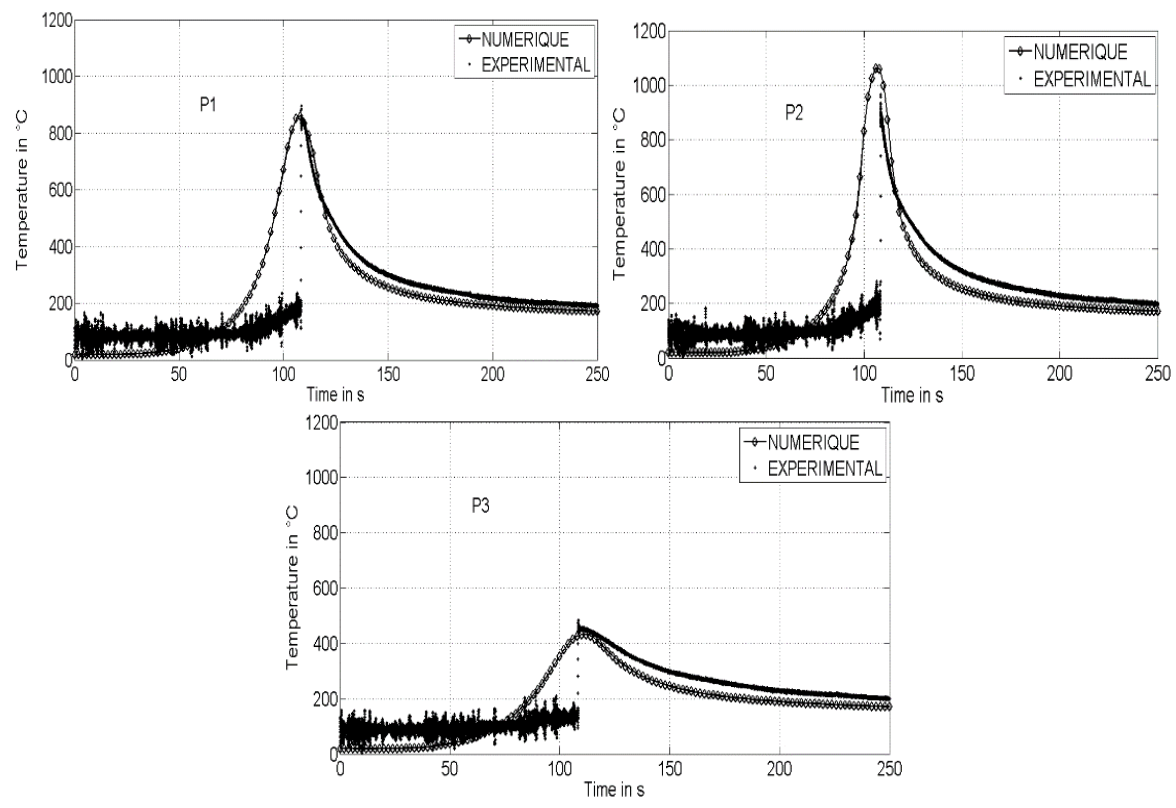

Figure 12 Comparison of numerical and experimental temperature evolutions during welding for speed welding $\mathrm{v}=0.72 \mathrm{~mm} / \mathrm{s}$ 
According to this figure, the comparison of the finite element simulated temperature evolution and those measured by thermocouples reveals a satisfactory agreement between numerical and experimental results. The same thermal behavior is noted during cooling step. However, during the heating phase, the difference between the measured values and those obtained by numerical simulation is due to the electrical disturbances induced by the welding electric arc which have an influence on thermocouples acquisition.

\section{CONCLUSION}

In the work, we have developed a computer finite element code using MATLAB software. This code aims to predict thermal behavior during C50-steel TIG-welding. The suggested model gives the possibility to determinate the space-time temperature evolutions in each point of the welded sheet from the beginning of welding until the return to thermal balance. In addition to numerical study, experimental investigations are made in order to measure temperature evolutions according to time during the welding of a $7 \mathrm{~mm}$ sheet. Comparison of numerical and experimental results shows a good consistency. The validated temperature fields at various time steps, resulting from the developed model, are used as an input data on the mechanical and metallurgical behavior simulation models during the TIG welding of C50 steel. Results of these models will be published subsequently.

\section{REFERENCES}

Aissani, M., Guessasma, S., Zitouni, A., Hamzaoui, R., Bassir, D.,Benkedda, Y., 2015. ThreeDimensional Simulation of 304L Steel TIG Welding Process: Contribution of The Thermal Flux. Applied Thermal Engineering,Volume 89, pp. 822-832

Anca, A., Cardona, A., Risso, J.,Fachinotti, V.D., 2011. Finite Element Modeling of Welding Processes. Applied Mathematical Modelling, Volume 35(2), pp. 688-707

Baskoro, A.S., Masuda, R., Suga, Y., 2011. Comparison of Particle Swarm Optimization and Genetic Algorithm for Molten Pool Detection in Fixed Aluminum Pipe Welding. International Journal of Technology, Volume 2(1), pp. 74-83

Belhadj, A., Bessrour, J., Masse, J.E., Bouhafs, M.,Barrallier, L., 2010. Finite Element Simulation of Magnesium Alloys Laser Beam Welding. Journal of Materials Processing Technology, Volume 210(9), pp. 1131-1137

Chuaiphan, W., Srijaroenpramong, L., 2018. Optimization of Gas Tungsten Arc Welding Parameters for the Dissimilar Welding between AISI 304 and AISI 201 Stainless Steels. Defence Technology, Volume 15(2), pp. 170-178

Del Coz Diaz, J.J., Rodríguez, P.M., Nieto, P.G., Castro-Fresno, D., 2010. Comparative Analysis of TIG Welding Distortions between Austenitic and Duplex Stainless Steels by FEM. Applied Thermal Engineering, Volume 30(16), pp. 2448-2459

Delmas, J., 2013. Functions of Form and Points of Integration Finite Elements. EDF Research and Development, GNU FDL

Depradeux, L., Jullien, J.F., 2004. 2D and 3D Numerical Simulations of TIG Welding of a 316L Steel Sheet. Revue Européenne des Eléments, Volume13(3-4),pp. 269-288

Ganesh, K.C., Vasudevan, M., Balasubramanian, K.R., Chandrasekhar, N., Mahadevan, S., Vasantharaja, P.,Jayakumar, T., 2014. Modeling, Prediction and Validation of Thermal Cycles, Residual Stresses and Distortion in Type 316 LN Stainless Steel Weld Joint Made by TIG Welding Process. Procedia Engineering, Volume 86, pp. 767-774

Hamide, M.,Bellet, M., 2007. Adaptive Anisotropic Mesh Technique for Coupled Problems: Application to Welding Simulation. In J.M. Cesar de Sa, \& A.D. Santos (Eds.), AIP Conference Proceedings, Volume 908(1), pp. 1561-1566 
Hamide, M., Massoni, E.,Bellet, M., 2008. Adaptive Mesh Technique for Thermal-Metallurgical Numerical Simulation of Arc Welding Processes. International Journal for Numerical Methods in Engineering, Volume 73(5), pp. 624-641

Lundbäck, A., 2003. Finite Element Modeling and Simulation of Welding of Aerospace Components. Doctoral Dissertation, Luleåtekniskauniversitet, Swedia

Seleš, K., Perić, M., Tonković, Z.,2018. Numerical Simulation of a Welding Process using a Prescribed Temperature Approach. Journal of Constructional Steel Research, Volume 145, pp. 49-57

Weiner, J.H., Boley, B.A., 1963. Elasto-Plastic Thermal Stresses in a Solidifying Body. Journal of the Mechanics and Physics of Solids, Volume11(3), pp. 145-154 